\title{
The short form endometriosis health profile (EHP-5): translation and validation study of the Iranian version
}

\author{
Azita Goshtasebi ${ }^{1}$, Maryam Nematollahzadeh ${ }^{1}$, Fatemeh Zahra Hariri ${ }^{1}$ and Ali Montazeri ${ }^{*}$
}

\begin{abstract}
Background: Endometriosis Health Profile (EHP-5) is a valid instrument to measure health-related quality of life in endometriosis. This study was conducted to culturally adapt and validate the EHP-5 in Iran.

Methods: Using a standard "forward-backward" translation procedure, the English language version of the questionnaire was translated into Persian (Iranian language). Then a sample of 199 women aged 18-50 years completed the questionnaire. To test reliability the internal consistency was assessed by Cronbach's alpha coefficient. Validity was evaluated using known groups comparison.
\end{abstract}

Results: The mean age of respondents was $31.4(\mathrm{SD}=5.4)$ years. Reliability analysis showed satisfactory result (Cronbach's alpha coefficient $=0.71$ ). The questionnaire discriminated well between sub-groups of women differing in infertility and premenstrual syndrome (PMS) in the expected direction.

Conclusion: This preliminary validation study of the Iranian version of the EHP-5 proved that it is an acceptable, reliable and valid measure of quality of life in endometriosis patients.

\section{Background}

Endometriosis is defined as the presence of endometrial tissue (gland and struma) outside the uterus. The most frequent sites of implantation are the pelvic viscera and the peritoneum. Endometriosis is one of the most common chronic gynecological conditions that significantly affect $10-15 \%$ of women of reproductive age $[1,2]$. Typically a woman with endometriosis will experience various symptoms including chronic pelvic pain, dyspareunia and dysmenorrhea, dysuria, abnormal uterine bleeding or spotting and sub-fertility [3] and that endometriosis represents a serious risk factor for developing ovarian cancer [4].

Several studies have indicated that endometriosis could affect psychological and social well-being and thus lead to significant reduction in health-related quality of life (HRQoL) [5]. Disease-specific instruments are developed to assess the aspects of quality of life particularly affected by a disease or disorder [6].

\footnotetext{
* Correspondence: montazeri@acecr.ac.ir

${ }^{2}$ Mental Health Research Group, Mother and Child Health Research Centre,

Iranian Institute for Health Sciences Research, ACECR, Tehran, Iran

Full list of author information is available at the end of the article
}

Recently Jones et al. developed the Endometriosis Health Profile-30 (EHP-30) that is the first standardized, new disease-specific instrument evaluating the health-related quality of life in women with endometriosis [7]. The EHP-30 questionnaire contains a core questionnaire with 30 items and five scales: pain, feeling of control and powerlessness, emotional well-being, social support, and self-image. Six modular parts including 23 questions were also provided to measure the areas of sexual intercourse, work, and relationship with children, feelings about medical profession, treatment and infertility [8]. The authors of EHP-30 decided to produce a shorter form of the questionnaire. It might be less time consuming and more practical, easy to interpret information obtained by instrument, easier for respondents to complete short questionnaire than EHP-30. The Endometriosis Health Profile-5 (EHP-5) was developed as a short version of the original questionnaire [9]. The aim of this study was to develop and validate the Iranian version of EHP-5. There was no such an instrument available in Iran.

\section{Biomed Central}




\section{Methods}

\section{Translation and culture adaptation}

Forward-backward procedure was applied to translate the English version of the EHP-5 into Persian (the Iranian language). Two independent professional translators produced two forward translations. Both translators were instructed to aim for conceptual rather than literal translation. Translators with one of the authors compared their translations and produced a single provisional version. Then two other professional translators translated the provisional questionnaire back into the English. The two translators were not aware of the questionnaire. Finally, an expert committee consisting of translators, the researchers, two midwifes, and one gynecologist and one epidemiologist reviewed all the translation and cultural adaptation processes were applied. They also evaluated the final English backward version with the original questionnaire. Consensus in terms of semantic, idiomatic, experiential, and conceptual equivalence was reached and a final version of the questionnaire (the Persian EHP-5) was provided. The final translated version of the questionnaire was given to 10 patients to complete and declare their understanding of the items to ensure face validity.

\section{Questionnaire}

The EHP-5 contains 11 questions (items): five items including pain, control and powerlessness, emotional well-being, lack of social support, self image from the core questionnaire and six items from the modular questionnaire that may not be applicable to every woman with endometriosis including work, intercourse, and worries about infertility, treatment, and relationship with children and medical professionals. Each item is rated on a four-point scale (never $=0$, rarely $=1$, sometimes $=2$, often $=3$, always $=4$ and not relevant if not applicable). Scores on the EHP-5 core and modular questionnaire then are transformed on a scale of 0 (indicating best possible health status) to 100 (indicating worst possible health status). If the 'not relevant' box was ticked for items on modular questionnaire the score could not be computed for that dimension.

\section{Sample and data collection}

The final draft of the Iranian version of the EHP-5 was administrated to a sample of 199 women with a confirmed surgical diagnosis of endometriosis undergoing conservative surgery. All women were selected from two obstetrics and gynecology clinics in Tehran, Iran (Royan Institute and Avicenna Research Institute both affiliated to Iranian Academic Center for Education, Culture and Research).
The sample size calculation was based on an assumption that at least $10 \%$ of women in the reproductive age would suffer from endometriosis. Two trained female midwifes collected the data by face-to-face interview 1 to 12 months after diagnostic laparoscopy. All patients completed a questionnaire containing brief background information (such as age, marital and reproductive status, and family history) and the EHP-5 questionnaire. The study was carried out during July 2009 to March 2011.

\section{Statistical analysis}

Internal consistency was assessed by calculating Cronbach's alpha coefficient. Value of 0.7 or greater was considered satisfactory [10]. Validity was assessed using known groups comparison to test how well the questionnaire discriminates between subgroups of the study sample that differed in reproductive health status. It was expected that women with infertility and PMS would have higher scores than women without infertility and PMS in all measures. Mann-Whitney U test was performed for comparisons. Women with infertility and PMS were identified after a confirmed diagnosis by gynecologists.

\section{Ethics}

The study received ethical approval from the Iranian Institute for Health Sciences Research. The authors informed all women regarding the study objectives, and indicated that their participation is voluntary and they could withdraw at any time. Both oral and written instructions were given to patients to ensure that items were understood (i.e. there were no right or wrong answers to the questions and the participants should feel free and honestly state what they think), and the subjects were reassured about the confidentiality.

\section{Results}

\section{The study sample}

In all, 220 women were approached and 199 (90\%) agreed to be interviewed. The main reason for those who did not participate in the study was dislike. The mean age of the respondents was $31.4(\mathrm{SD}=5.4)$ years. Most were married (94.5\%) and university educated $(43.3 \%)$. The characteristics of the respondents are shown in Table 1.

\section{Descriptive statistics and reliability}

The descriptive statistics of the 5 items are shown in Table 2. The Cronbach's alpha coefficient was 0.71 for the instrument indicating a satisfactory result. 
Table 1 Demographic characteristics of the studied women $(n=199)$

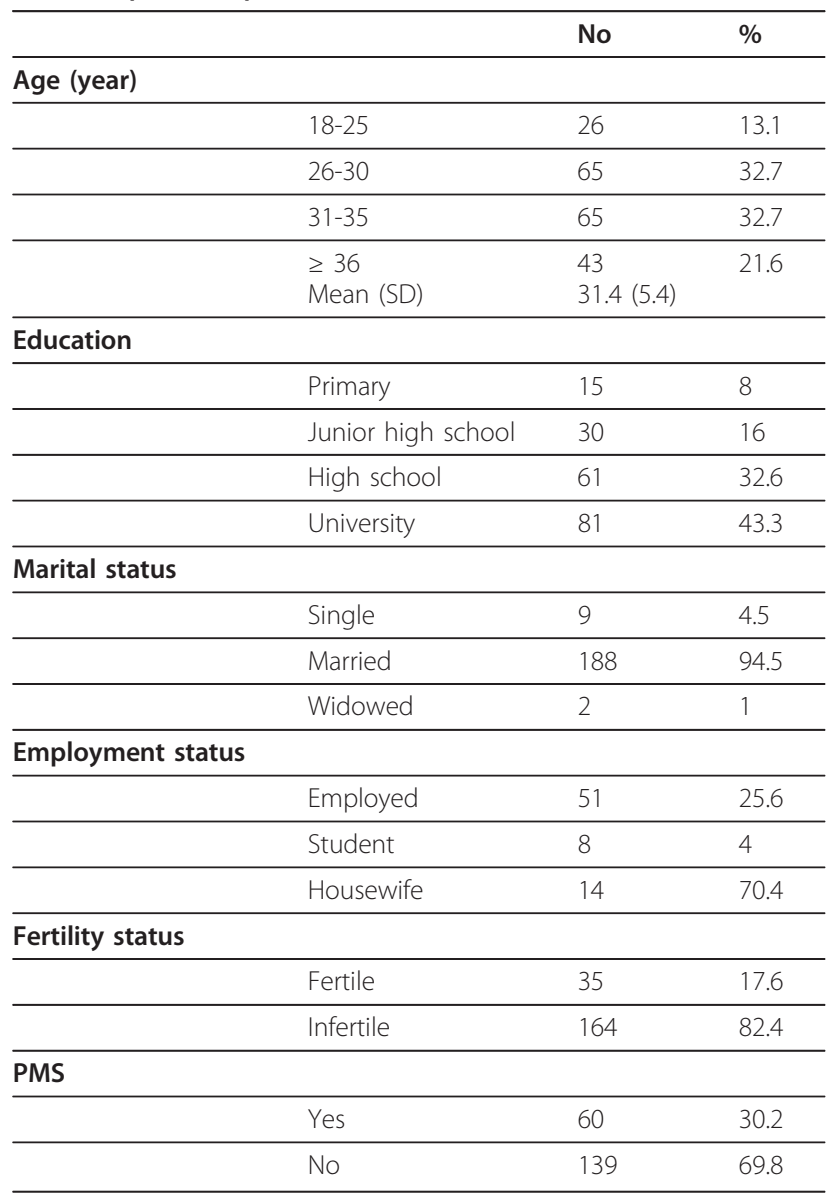

\section{Known groups comparison}

Known groups comparison was used to test the validity. It was hypothesized that women with infertility and PMS would have poorer quality of life than women without infertility and PMS. The analysis showed that the women with infertility had higher scores in pain, control and powerlessness, emotional well-being and self image and individuals suffering PMS had lower scores in pain, control and powerlessness, emotional well-being and lack of social support measures as expected (Table 3). This indicated that the EHP-5 well discriminated between subgroups of the people who differed in reproductive health status.

\section{Discussion}

Although cross-cultural validation studies are very difficult to be carried out, their results might be considered worthwhile. Firstly, they provide standard health measures that make health status comparisons between different populations possible. Secondly, they provide validated instrument to monitor population health, estimate burden of disease and investigate outcomes in clinical practice and evaluate treatment effects. This was the first study on psychometric properties of the Iranian version of EHP-5 among an Iranian population. The results showed that the instrument was a reliable and valid measure that can be used in monitoring and measuring health-related quality of life of women with endometriosis.

Similarly the validity of the EHP-5 in different cultures was well documented. For instance, the finding from an English study showed that the instrument had good validity and could be applied among women with endometriosis [9]. Furthermore, a French version of the EHP-5 questionnaire has been developed and its acceptability and feasibility was desirable although validity was not reported [10].

Iranian version of the EHP-5 was extracted from its English version. The translation of the EHP-5 in Iran went through a rigorous method and was approved by the questionnaire's developers. Thus we did not encounter any difficulties in data collection.

The EHP-5 was basically designed to be a self-administrated questionnaire but it can be completed through an interview in person or by telephone [11]. However, faceto-face administration of questionnaire allowed the interviewers to collect data without any missing data. Although method of completing the EHP-5 has not been mentioned in its manual, the designers administered it by mail (self-administrated) and the rate of returning the questionnaire was reported to be $37.1 \%$ [9].

Reliability was assessed by internal consistency and validity was examined by known groups comparison. Cronbach's alpha coefficient showed a satisfactory result

Table 2 Descriptive statistics for the EHP-5 core questionnaire

\begin{tabular}{|c|c|c|c|c|c|c|c|c|}
\hline & \multirow[t]{2}{*}{ Mean row scores (SD) } & \multirow[t]{2}{*}{$95 \% \mathrm{Cl}$} & \multirow[t]{2}{*}{ Skewness } & \multicolumn{5}{|c|}{ Response frequencies (\%) } \\
\hline & & & & Never & Rarely & Some times & Often & Always \\
\hline Pain & $0.085(0.07)$ & $0.7-1$ & 0.937 & 52.8 & 17.1 & 24.1 & 4 & 2 \\
\hline Control \& powerlessness & $1.44(0.09)$ & $1.26-1.62$ & -0.95 & 34.7 & 15.1 & 29.6 & 12.6 & 8 \\
\hline Emotional well-being & $1.68(1.3)$ & $1.50-1.87$ & 0.20 & 25.1 & 18.1 & 30.7 & 15.1 & 11.1 \\
\hline Lack of social support & $1.37(1.35)$ & $1.18-1.57$ & 0.511 & 38.7 & 16.6 & 22.1 & 13.6 & 9 \\
\hline Self image & $1.48(1.42)$ & $1.27-1.68$ & 0.375 & 40.2 & 12.6 & 17.1 & 19.1 & 11.1 \\
\hline
\end{tabular}


Table 3 known groups comparison for the EHP-5*

\begin{tabular}{llll}
\hline Infertility & No $(\mathbf{n}=\mathbf{3 5})$ & Yes $(\mathbf{n}=\mathbf{1 6 4})$ & \\
\hline & Mean (SEM) & Mean (SEM) & $\mathbf{P}^{* *}$ \\
\hline Pain & $19.05(2.01)$ & $32.14(4.29)$ & 0.007 \\
\hline Control \& powerlessness & $34.14(2.54)$ & $45.0(5.2)$ & 0.07 \\
\hline Emotional well-being & $38.87(2.39)$ & $57.85(6.21)$ & 0.002 \\
\hline Lack of social support & $32.85(5.95)$ & $34.75(2.62)$ & 0.7 \\
\hline Self image & $32.77(2.74)$ & $57.14(6.02)$ & $<0.0001$ \\
\hline PMS & No $(\mathbf{n}=139)$ & Yes $(\mathbf{n}=60)$ & \\
\hline Pain & Mean (SEM) & Mean (SEM) & $\mathbf{P} * *$ \\
\hline Control \& powerlessness & $31.47(2.60)$ & $46.66(4.38)$ & 0.002 \\
\hline Emotional well-being & $37.76(2.57)$ & $52.5(4.55)$ & 0.003 \\
\hline Lack of social support & $33.81(2.88)$ & $35.83(4.34)$ & 0.7 \\
\hline Self image & $33.45(2.97)$ & $45.41(4.91)$ & 0.03 \\
\hline
\end{tabular}

* The higher scores indicate worse conditions

** Derived from Mann-Whitney U test.

[12]. Known groups comparison indicated that the EHP5 score were able to distinguish very well between subgroups of the respondents who differed in reproductive health status. The study showed that women with infertility and PMS had poorer health compared to women without infertility and PMS. These findings are consistent with results from other studies carried out in different countries [12-15]. However, there were no significant differences in social support between fertile and infertile or women with and without PMS. This might be explained by the fact that endometriosis by itself is a chronic disease and thus as it relates to social support, both fertile and infertile women or those with and without PMS showed a relatively similar scores and therefore one might not expect to find significant differences between women in this domain.

Tools assessing quality of life are being used in research and clinical trials rarely. For instance, only $17 \%$ of randomized trials assessed in a systematic review on the measurement of HRQoL in women had used standardized instruments [15]. In addition, often the instruments have been used in research just measured one dimension of illness e.g. psychological health status without identifying other areas of well being affected by disease [16]. Reasons for the limited use of health statues instruments in clinical setting are that they are too long and complicated for clinicians to understand and interpret the data gained by long health statues instruments, and also they are too burdensome for respondent to complete them $[17,18]$. The short form EHP-5 provides the chance of using a very brief instrument that measures health outcome for women with endometriosis where the long form version would not be appropriate. The results obtained by the EHP- 5 from the analyses suggest this instrument provides the same picture of health-related quality of life as the longer version [9].

Although this study did not provide evidence for testretest reliability, responsiveness to change or other tests; overall the findings showed that the Iranian version of EHP-5 is a reliable measure for measuring health quality of life in endometriosis patients. It will be especially useful in clinical settings where a short and economical endometriosis health status measure is needed. The future studies could focus on other psychometric properties of the EHP-5 questionnaire and also on different applications of the questionnaire as a recent study has suggested even it is a useful index in order to evaluate cost-effectiveness of healthcare interventions [19].

\section{Conclusion}

This study presents the first step in evaluating psychometric properties of a well-known instrument measuring health-related quality of life of Iranian patients with endometriosis. Since health related quality of life was rarely assessed as primary end-point in studies of endometriosis in Iran, the Persian EHP-5 might possibly provide both clinicians and patients with numerous advantages as an important outcome measure in future studies. However, its sensitivity to change needs still to be studied.

\section{Acknowledgements}

We wish to express gratitude to people who co-operated in the selection of the patients and patients who gave their time to complete the questionnaire.

\section{Author details}

'Family Health Research Group, Mother and Child Health Research Centre, Iranian Institute for Health Sciences Research, ACECR, Tehran, Iran. ${ }^{2}$ Mental Health Research Group, Mother and Child Health Research Centre, Iranian Institute for Health Sciences Research, ACECR, Tehran, Iran.

\section{Authors' contributions}

All authors were involved in designing of the study, data collection and analysis, interpretation of results and manuscript preparation. AG, MN and FZH prepared the first draft of the paper. AM and AG provided the final manuscript. All authors read and approved the final manuscript.

\section{Competing interests}

The authors declare that they have no competing interests.

Received: 31 May 2011 Accepted: 27 July 2011 Published: 27 July 2011

\section{References}

1. Melin A, Sparén P, Persson I, Bergqvist A: Endometriosis and the risk of cancer with special emphasis on ovarian cancer. Human Reproduction 2006, 98:1237-42.

2. Berek JS: Berek and Novak's Gynecology Philadelphia: Lippincott Williams and Wilkins; 2007, 1137-1184

3. Gao X, Yeh YC, Outley J, simon J, Botteman M, Spalding J: Health-related quality of life burden of women with endometriosis: a literature review. Current Medical Research and Opinion 2006, 22:1787-1797.

4. Aris A: Endometriosis-associated ovarian cancer: A ten-year cohort study of women living in the Estrie Region of Quebec, Canada. J Ovarian Res 2010, 3:2. 
5. Lockhat FB, Emembolu JO, Konje JC: The evaluation of the effectiveness of an intrauterine administered progestogen (levonorgestrel) in the symptomatic treatment of endometriosis and in the staging of the disease. Human Reproduction 2004, 19:179-184.

6. Jones G, Jenkinson C, Kennedy S: Development of the Short Form Endometriosis Health Profile Questionnaire: The EHP-5. Quality of Life Research 2004, 13:695-704.

7. Jones G, Kennedy S, Barnard A, Wong J, Jenkinson C: Development of an endometriosis quality-of-life instrument: The Endometriosis Health Profile-30. Obstetrics \& Gynecology 2001, 98:258-264.

8. Jones G, Jenkinson C, Taylor N, Mills A, Kennedy S: Measuring quality of life in women with endometriosis: tests of data quality, score reliability, response rate and scaling assumptions of the Endometriosis Health Profile Questionnaire. Human Reproduction 2006, 21:2686-2693.

9. Renouvel F, Fauconnier A, Pilkington H, Panel P: Linguistic adaptation of the endometriosis health profile 5: EHP-5. Journal de Gynécologie Obstétrique et Biologie de la Reproduction 2009, 38:404-410.

10. Nunally JC, Bernstien IH: Psychometric theory New York: Mc Grow Hill; 1994

11. Denny E: Women's experience of endometriosis. Acta Obstet Gynecol Scand 2001, 80:628-637.

12. Gandek B, Ware JE: Methods for validating and norming translations of health status questionnaire: The IQoLA project approach. J Clin Epidemiol 1998, 51:953-959.

13. Levine $\mathrm{S}$ : The changing terrains in medical sociology: emergent concern with quality of life. J Health Soc Behav 1987, 28:1-6.

14. Wilson IB, Cleary PD: Linking clinical variables with health related quality of life. A conceptual model of patient outcomes. J Am Med Assoc 1995, 273:59-65.

15. Jones GL, Kennedy SH, Jenkinson C: Health-related quality of life measurement in women with common benign gynecologic conditions: a systematic review. Am J Obstet Gynecol 2002, 187:501-511.

16. Fitzpatrick R: Applications of health status measures. In Measuring Health and Medical Outcomes. Edited by: Jenkinson C. London: UCL Press; 1994:27-41.

17. Coste J, Guillemin F, Pouchet J, Fermanian J: Methodological approaches to shortening composite measurement scales. J Clin Epidemiol 1997, 50:247-252.

18. Cox DR, Fitzpatrick R, Fletcher $A E$, Gore $S M$, Spiegelhalter $D J$, Jones $D R$ : Quality of life assessment: can we keep it simple? J R Statist Soc A 1992 155:353-393.

19. Winkel CA: A cost-effective approach to the management of endometriosis. Curr Opin Obstet Gynecol 2000, 12:317-320.

doi:10.1186/1757-2215-4-11

Cite this article as: Goshtasebi et al:: The short form endometriosis health profile (EHP-5): translation and validation study of the Iranian version. Journal of Ovarian Research 2011 4:11.

\section{Submit your next manuscript to BioMed Central and take full advantage of:}

- Convenient online submission

- Thorough peer review

- No space constraints or color figure charges

- Immediate publication on acceptance

- Inclusion in PubMed, CAS, Scopus and Google Scholar

- Research which is freely available for redistribution

Submit your manuscript at www.biomedcentral.com/submit 